\title{
Antimicrobial screening of synthesized some novel azomethines via organic base
}

\author{
G. R. Patel, J. J. Maru*, Rakesh Yadav \\ Department of Chemistry, Sheth M. N. Science College, NGES Campus, \\ Patan - 384265, India \\ *E-mail address: jay1maru@yahoo.in
}

\begin{abstract}
The author has synthesized azomethines in which carbonyl group is replaced by amine of acid hydrazides. The intermediates were prepared by using 2-mercapto benzimidazole with N-(4-Acetylphenyl)-2-chloro-acetamide in the presence of $\mathrm{K}_{2} \mathrm{CO}_{3}$. The Resulted product was further treated with different substituted of acid hydrazide in ethanol to yield the titled compounds using a catalytic amount of acetic acid. New products were examined for their antibacterial effects against Grampositive and Gram-negative strains and antifungal were highly potent with lowest MIC Values. The structural assignments of the new products were done on the basis of FT-IR, NMR, Mass spectroscopy and elemental analysis.
\end{abstract}

Keywords: Azomethine; Acid hydrazide; Benzimidazole; Antimicrobial activity

\section{INTRODUCTION}

Development of antimicrobial agents for the chemotherapy of fungal and bacterial infections represents one of the most significant achievements of this decade. The rising emergence of acquired resistance to existing antimicrobials to fungal and bacterial infections leads us to synthesize newer antimicrobial agents. Benzimidazole nucleus is found in many clinically useful chemotherapeutic agents [1] shown by literature survey. The versatility of benzimidazole and its potential to yield derivatives with a wide range of biological activities has made it a useful structure for further molecular exploration. The substituents at 1- and 2positions of this heterocycle have been intensively studied and this led to derivatives with good antimicrobial activity. It is well known that several 1,2-disubstituted benzimidazoles [2,3] are potent inhibitors of RNA viruses and this effect encouraged intensive efforts to prepare and evaluate other derivatives. 2-Mercaptobenzimidazole is derivative of benzimidazole exhibited a wide variety of interesting biological activities such as antimicrobial [4], anticonvulsant [5], antidiabetic [5], antiulcer agent [6], Antibacterial [7] and antifungal [7], etc. Aceto group containing benzimidazole which reacts with different amine produced azomethine moiety [8]. 
Azomethine are products of condensation of simple or substituted acid hydrazide with simple or substituted Aceto group containing compound in the presence of catalytic amount of acetic acid. Azomethine constitute an important group of natural products and some of them possess a wide range of biological activities such as Antifungal [9], anti-inflammatory [10], anti-bacterial [11], antioxidant [12] etc.

\section{EXPERIMENTAL}<smiles>CC(=O)c1ccc(N)cc1</smiles><smiles>CC(=O)c1ccccc1</smiles><smiles>Ic1ccccc1</smiles><smiles>NCC(=O)Cl</smiles><smiles>C1CCCCC1</smiles><smiles>Sc1nc2ccccc2[nH]1</smiles>

2

$$
\begin{gathered}
\text { K2CO3 /Acetone } \\
\text { Stirring at RTR } \\
4 \text { hrs }
\end{gathered}
$$<smiles>CNc1ccc(C(C)=O)cc1</smiles><smiles>C=C(I)CC</smiles><smiles>CSc1nc2ccccc2[nH]1</smiles>

4<smiles>[R]c1cccc(C(=O)NN)c1</smiles><smiles>[R]c1ccc(C(=O)N/N=C(\C)c2ccc(NC(=O)CSc3nc4ccccc4[nH]3)cc2)cc1</smiles>

5a-1

Where $\mathbf{R}=$ different substituents

Scheme 1. Synthetic route for the preparation of title compounds 5a-1.

All the required compounds and solvents for the synthesis were used from Merck Ltd., LOBA Chemie and sdfine chemicals. Melting point was determined by the open end capillary method and are reported uncorrected. Completion of reaction was monitored by aluminum coated TLC plates (TLC silica gel 60 F245, E. Merck) using n-hexane: ethyl acetate (7.5:2.5 
$\mathrm{V} / \mathrm{V}$ ) as mobile phase and spot checked under ultraviolet (UV) light. Bruker Spectrophotometer-400 MHz where DMSO-d6 was used as solvent and TMS as Reference used for the 1H NMR and 13C NMR. Shimadzu mass Spectrophotometer used for the Mass spectral analysis. FT-IR alpha-t (ATR) used for the IR spectral data. Perkin-Elmer $2400 \mathrm{CHN}$ Analyzer used for the elemental analysis $(\% \mathrm{C}, \mathrm{H}, \mathrm{N})$.

General procedure for the synthesis of the title compounds 5a-1: Compound 4, 2(1Hbenzo[d]imidazol-2-ylthio)-N-(4-acetylphenyl)acetamide $(0.01 \mathrm{~mol}, 325 \mathrm{gm} / \mathrm{mol}, 3.25 \mathrm{gm})$ was further treated with different substituted acid hydrazide $(0.01 \mathrm{~mol})$ in ethanol $(20 \mathrm{ml})$ in the presence of catalytic amount of acetic acid and refluxed for 8 hours. Resulting solid was separated out, filtered, and washed with water and dried and recrystallized by alcohol (99.9\%) [13] [14]. The products were designated as $5 \mathrm{a}-1$ and characterized by elemental, IR, NMR, CMR and MS analyses.

\section{1. Physical constant and characterization of $2-(1 \mathrm{H}-$-benzo[d]imidazole-2-ylthio)-N-(4- acetylphenyl)acetamide (4)}

Yield: $82 \%$; M.P.: $168-170{ }^{\circ} \mathrm{C}$; IR $\left(v_{\max }, \mathrm{cm}^{-1}\right.$, ATR): $1384\left(\mathrm{C}-\mathrm{H}\right.$ bending $\left.-\mathrm{CH}_{3}\right), 1587$ $(\mathrm{C}=\mathrm{C}$ stretching, aromatic ring $), 1677\left(\mathrm{C}=\mathrm{O}\right.$ stretching $\left.-\mathrm{COCH}_{3}\right), 1697(\mathrm{C}=\mathrm{O}$ stretching CONH-), 2937 (C-H stretching $-\mathrm{CH}_{3}$ ), 3050 (C-H stretching, aromatic ring); 1H NMR (400 MHz, DMSO- $\left.\delta_{6}, \delta, \mathrm{ppm}\right): 2.55\left(3 \mathrm{H}, \mathrm{s},-\mathrm{CH}_{3}\right), 3.89\left(2 \mathrm{H}, \mathrm{s},-\mathrm{CH}_{2}\right), 7.20-7.94(8 \mathrm{H}, \mathrm{d}, \mathrm{Ar}-\mathrm{H})$, 5.00-7.21 (2H, s, -NH); 13C NMR (400 MHz, DMSO- $\left.\delta_{6}, \delta, \mathrm{ppm}\right): 23.2,38.28,115.6,116.8$, 121.3, 121.3, 124.2, 124.2, 128.1, 128.1, 135.1, 141.6, 141.6.0, 143.2, 143.2, 168.3 , 196.6 ; MS (m/z): $326\left(\mathrm{M}^{+}\right)$; Elemental Analysis: C, 62.62; H, 4.34; N, 12.92\%.

\section{2. Physical constant and characterization of $2-(1 \mathrm{H}-$-benzo[d]imidazole-2-ylthio)-N-(4- (1-(2-benzoylhydrazono)ethyl)phenyl) acetamide (5a)}

Yield: $70 \%$; M.P.: $192-194{ }^{\circ} \mathrm{C}$; $1 \mathrm{H}$ NMR (400 MHz, DMSO- $\delta 6, \delta$, ppm): 2.29 (3H, s, $\left.\mathrm{CH}_{3}\right), 4.08\left(2 \mathrm{H}, \mathrm{s},-\mathrm{CH}_{2}\right), 7.20-8.09(12 \mathrm{H}, \mathrm{d}, \mathrm{Ar}-\mathrm{H}), 5.23-7.12(2 \mathrm{H}, \mathrm{s},-\mathrm{NH}) ; 13 \mathrm{C}$ NMR (400 MHz, DMSO- $\delta 6, \delta$, ppm): 16.8, 39.2, 113.9, 113.9, 119.8,119.8, 121.2, 121.2, 125.9, 125.9, $128.2,128.2,129.0,129.0,131.5,132.5,132.5,137.6,137.6,139.5,147.5,147.5,163.9$, 166.7 ; MS (m/z): $443\left(\mathrm{M}^{+}\right)$; Elemental Analysis: C, 65.23; H, 4.80; N, 15.88\%.

\section{3. Physical constant and characterization of 2 -(1H-benzo[d]imidazole-2-ylthio)-N-(4- (1-(2-(3-nitrobenzoyl)hydrazono)ethyl)phenyl) acetamide (5b)}

Yield: $75 \%$; M.P.: $210-212{ }^{\circ} \mathrm{C}$; $1 \mathrm{H}$ NMR (400 MHz, DMSO- $\left.\delta 6, \delta, \mathrm{ppm}\right): 2.34(3 \mathrm{H}, \mathrm{s},-$ $\left.\mathrm{CH}_{3}\right), 4.10\left(2 \mathrm{H}, \mathrm{s},-\mathrm{CH}_{2}\right), 7.20-8.75(12 \mathrm{H}, \mathrm{d}, \mathrm{Ar}-\mathrm{H}), 5.48-7.22(2 \mathrm{H}, \mathrm{s},-\mathrm{NH}) ; 13 \mathrm{C}$ NMR (400 MHz, DMSO- $\delta 6, \delta$, ppm): 16.4, 38.8, 113.1, 113.1, 118.9, 121.6, 121.6, 122.1, 126.1, 126.1, $127.9,129.5,129.5,132.1,132.1,133.0,138.0,138.0,140.1,145.9,148.3,148.3,163.7$, 166.3 ; MS (m/z): $489\left(\mathrm{M}^{+}\right)$; Elemental Analysis : C, 59.23; H, 4.22; N, 17.24\%.

\section{4. Physical constant and characterization of 2 -(1H-benzo[d]imidazole-2-ylthio)-N-(4- (1-(2-(4-nitrobenzoyl)hydrazono)ethyl)phenyl) acetamide (5c)}

Yield: $83 \%$; M.P.: $212-214{ }^{\circ} \mathrm{C}$; $1 \mathrm{H}$ NMR (400 MHz, DMSO- $\delta 6, \delta$, ppm): 2.33 (3H, s, $\left.\mathrm{CH}_{3}\right), 4.05$ (2H, s, $\left.-\mathrm{CH}_{2}\right), 7.20-8.44(12 \mathrm{H}, \mathrm{d}, \mathrm{Ar}-\mathrm{H}), 4.92-7.12(2 \mathrm{H}, \mathrm{s},-\mathrm{NH}) ; 13 \mathrm{C}$ NMR (400 MHz, DMSO- $\delta 6, \delta$, ppm): 16.7, 39.5, 113.9, 113.9, 120.8, 120.8, 121.7, 121.7, 125.0, 128.9, $128.9,129.2,129.2,131.0,132.4,132.4,137.6,137.6,140.1,145.4,147.5,147.5,163.2$, 166.7 ; MS (m/z): $488\left(\mathrm{M}^{+}\right)$; Elemental Analysis: C, 59.13; H, 4.12; N, 17.42\%. 
2. 5. Physical constant and characterization of $2-(1 \mathrm{H}-b e n z o[d]$ imidazole-2-ylthio)-N-(4(1-(2-(3-methylbenzoyl)hydrazono)ethyl)phenyl) acetamide (5d)

Yield: $62 \%$; M.P.: $200-202{ }^{\circ} \mathrm{C}$; $1 \mathrm{H}$ NMR (400 MHz, DMSO- $\left.\delta 6, \delta, \mathrm{ppm}\right): 2.30-2.36(6 \mathrm{H}$, s, $-\mathrm{CH} 3), 4.08\left(2 \mathrm{H}, \mathrm{s},-\mathrm{CH}_{2}\right), 7.22-7.90(12 \mathrm{H}, \mathrm{d}, \mathrm{Ar}-\mathrm{H}), 5.24-7.12(2 \mathrm{H}, \mathrm{s},-\mathrm{NH}) ; 13 \mathrm{C}$ NMR (400 MHz, DMSO- $\delta 6, \delta$, ppm): 16.2, 21.6, 38.9, 113.2, 113.2, 120.4, 120.4, 122.3, 122.3, $124.5,128.8,128.8,129.6,129.6,131.5,131.5,132.6,137.9,137.9,139.0,139.0,145.9$, 147.4, 163.4, 167.2; MS (m/z): $457\left(\mathrm{M}^{+}\right)$; Elemental Analysis: C, 65.66; H, 5.14; N, 15.36\%.

2. 6. Physical constant and char acterization of 2 -(1H-benzo[d]imidazole-2-ylthio)-N-(4(1-(2-(4-methylbenzoyl)hydrazono)ethyl)phenyl) acetamide (5e)

Yield: $65 \%$; M.P.: $208-210{ }^{\circ} \mathrm{C}$; $1 \mathrm{H}$ NMR (400 MHz, DMSO- $\left.\delta 6, \delta, \mathrm{ppm}\right): 2.30-2.35(6 \mathrm{H}, \mathrm{s}$, $\left.-\mathrm{CH}_{3}\right), 4.10\left(2 \mathrm{H}, \mathrm{s},-\mathrm{CH}_{2}\right), 7.20-7.85(12 \mathrm{H}, \mathrm{d}, \mathrm{Ar}-\mathrm{H}), 5.22-6.98(2 \mathrm{H}, \mathrm{s},-\mathrm{NH}) ; 13 \mathrm{C}$ NMR (400 MHz, DMSO- $\delta 6, \delta, \mathrm{ppm}$ ): 16.4, 22.2, 38.2, 114.2, 114.2, 119.9, 119.9, 121.2, 121.2, $125.7,125.7,128.8,128.8,129.7,129.7,131.2,133.7,133.7,137.8,137.8,139.3,146.4$, 146.4, 163.5, 166.6 ; MS (m/z): $459\left(\mathrm{M}^{+}\right)$; Elemental Analysis: C, 65.45; H, 5.22; N, 15.32\%.

2. 7. Physical constant and characterization of $2-(1 \mathrm{H}-b e n z o[d] i m i d a z o l e-2-y l t h i o)-N-(4-$ (1-(2-(4-methoxybenzoyl)hydrazono)ethyl)phenyl) acetamide (5f)

Yield: $59 \%$; M.P.: $218-220{ }^{\circ} \mathrm{C}$; $1 \mathrm{H}$ NMR (400 MHz, DMSO- $\delta 6, \delta$, ppm): 2.29 (3H, s, $\left.\mathrm{CH}_{3}\right), 4.08\left(2 \mathrm{H}, \mathrm{s},-\mathrm{CH}_{2}\right), 7.20-8.09(12 \mathrm{H}, \mathrm{d}, \mathrm{Ar}-\mathrm{H}), 7.22(1 \mathrm{H}, \mathrm{s},-\mathrm{NH}) ; 13 \mathrm{C}$ NMR (400 MHz, DMSO- $\delta 6, \delta$, ppm): 16.8, 39.2, 113.8, 113.8, 119.8, 119.8, 121.3, 121.3, 125.9, 126.5, $128.2,128.2,129.0,129.0,132.2,132.2,132.5,137.6,137.6,139.5,146.6,146.6,163.9$, 166.7; MS (m/z): $474\left(\mathrm{M}^{+}\right)$; Elemental Analysis: C, 63.53; H, 4.95; N, 14.98\%.

2. 8. Physical constant and characterization of $2-(1 \mathrm{H}-b e n z o[d] i m i d a z o l e-2-y l t h i o)-N-(4-$ (1-(2-(2-chlorobenzoyl)hydrazono)ethyl)phenyl) acetamide (5g)

Yield: $73 \%$; M.P.: $214-216{ }^{\circ} \mathrm{C}$; $1 \mathrm{H}$ NMR (400 MHz, DMSO- $\delta 6, \delta$, ppm): $2.32(3 \mathrm{H}$, s, $\left.\mathrm{CH}_{3}\right), 4.10\left(2 \mathrm{H}, \mathrm{s},-\mathrm{CH}_{2}\right), 7.20-7.90(12 \mathrm{H}, \mathrm{d}, \mathrm{Ar}-\mathrm{H}), 5.12-7.12(2 \mathrm{H}, \mathrm{s},-\mathrm{NH}) ; 13 \mathrm{C}$ NMR (400 MHz, DMSO- $\delta 6, \delta$, ppm): 16.8, 39.4, 113.8, 113.8, 119.8, 119.8, 121.6, 121.6, 125.9, 125.9, $129.0,129.0,129.6,131.0,132.6,132.6,137.6,137.6,139.5,139.5,146.0,146.0,163.0$, 166.4 ; MS (m/z): $479(\mathrm{M}+)$; Elemental Analysis: C, 61.12; H, 4.26; N, 14.88\%.

\section{9. Physical constant and characterization of $2-(1 \mathrm{H}-$ benzo[d]imidazole-2-ylthio)-N-(4-} (1-(2-(3-chlorobenzoyl)hydrazono)ethyl)phenyl) acetamide (5h)

Yield: $70 \%$; M.P.: $214-216{ }^{\circ} \mathrm{C}$; $1 \mathrm{H}$ NMR (400 MHz, DMSO- $\delta 6, \delta$, ppm): $2.30(3 \mathrm{H}, \mathrm{s},-$ $\left.\mathrm{CH}_{3}\right), 4.06\left(2 \mathrm{H}, \mathrm{s},-\mathrm{CH}_{2}\right), 7.22-7.88(12 \mathrm{H}, \mathrm{d}, \mathrm{Ar}-\mathrm{H}), 5.04-7.00(2 \mathrm{H}, \mathrm{s},-\mathrm{NH}) ; 13 \mathrm{C}$ NMR (400 MHz, DMSO- $\delta 6, \delta$, ppm): 16.8, 39.4, 113.4, 113.4, 118.6, 118.6, 121.3, 121.3, 125.9, 125.9, $129.0,129.0,131.6,131.6,132.5,132.5,137.4,137.4,139.6,139.6,146.8,148.2,163.5$, 166.0 ; MS (m/z): $479\left(\mathrm{M}^{+}\right)$; Elemental Analysis: C, 61.21; H, 4.14; N, 14.86\%.

2. 10. Physical constant and characterization of $2-(1 \mathrm{H}-$ benzo[d]imidazole-2-ylthio)-N-(4(1-(2-(4-chlorobenzoyl)hydrazono)ethyl)phenyl) acetamide (5i)

Yield: $70 \%$; M.P.: $216-218{ }^{\circ} \mathrm{C}$; $1 \mathrm{H}$ NMR (400 MHz, DMSO- $\delta 6, \delta$, ppm): $2.30(3 \mathrm{H}, \mathrm{s},-$ $\left.\mathrm{CH}_{3}\right), 4.02\left(2 \mathrm{H}, \mathrm{s},-\mathrm{CH}_{2}\right), 7.22-8-04(12 \mathrm{H}, \mathrm{d}, \mathrm{Ar}-\mathrm{H}), 5.00-7.20(2 \mathrm{H}, \mathrm{s},-\mathrm{NH})$; 13C NMR (400 MHz, DMSO- $\delta 6, \delta$, ppm): 16.9, 39.4, 113.9, 113.9, 119.0, 119.0, 121.8, 121.8, 126.8, 128.0, 
$128.0,129.6,129.6,131.5,132.5,132.5,137.8,137.8,139.6,139.6,146.6,146.6,163.6$, 166.2 ; MS (m/z): $477\left(\mathrm{M}^{+}\right)$; Elemental Analysis: C, 60.44; H, 4.23; N, 14.62\%.

2. 11. Physical constant and characterization of $2-(1 \mathrm{H}-$-benzo[d]imidazole-2-ylthio)-N-(4(1-(2-(2-hydroxybenzoyl)hydrazono)ethyl)phenyl) acetamide (5j)

Yield: $64 \%$; M.P.: $222-224{ }^{\circ} \mathrm{C}$; $1 \mathrm{H}$ NMR (400 MHz, DMSO- $\delta 6, \delta$, ppm): 2.24 (3H, s, $\left.\mathrm{CH}_{3}\right), 5.31(1 \mathrm{H}, \mathrm{s},-\mathrm{OH}), 4.05\left(2 \mathrm{H}, \mathrm{s},-\mathrm{CH}_{2}\right), 7.14-7.78(12 \mathrm{H}, \mathrm{d}, \mathrm{Ar}-\mathrm{H}), 4.90-7.12(2 \mathrm{H}, \mathrm{s},-$ $\mathrm{NH}$ ); $13 \mathrm{C}$ NMR (400 MHz, DMSO- $\delta 6, \delta, \mathrm{ppm}): 16.8,39.4,113.8,113.9,116.0,116.0$, $119.8,119.8,121.4,121.4,125.9,126.5,128.2$, 128.2, 131.2, 132.1, 132.5, 137.6, 137.6, 139.5, 146.6, 147.5, 163.9, 166.7 ; MS (m/z): $459\left(\mathrm{M}^{+}\right)$; Elemental Analysis: C, 58.68; H, $4.38 ; \mathrm{N}, 16.38 \%$.

2. 12. Physical constant and characterization of $2-(1 \mathrm{H}-$ benzo[d]imidazole-2-ylthio)-N-(4(1-(2-(3-hydroxybenzoyl)hydrazono)ethyl)phenyl) acetamide (5k)

Yield: $61 \%$; M.P.: $226-228^{\circ} \mathrm{C}$; $1 \mathrm{H}$ NMR (400 MHz, DMSO- $\delta 6, \delta$, ppm): $2.26(3 \mathrm{H}, \mathrm{s},-$ $\left.\mathrm{CH}_{3}\right), 5.25(1 \mathrm{H}, \mathrm{s},-\mathrm{OH}), 4.13\left(2 \mathrm{H}, \mathrm{s},-\mathrm{CH}_{2}\right), 7.08-7.75(12 \mathrm{H}, \mathrm{d}, \mathrm{Ar}-\mathrm{H}), 5-12-76-90(2 \mathrm{H}, \mathrm{s},-$ $\mathrm{NH})$; 13C NMR (400 MHz, DMSO- $\delta 6, \delta, \mathrm{ppm})$ : 16.6, 39.4, 113.9, 113.9, 116.4, 116.4, $119.8,119.8,121.2,121.2,125.9,125.9,129.2,129.2,131.2,132.5,132.5,137.6,137.6$, 139.5, 147.5, 147.5, 163.5, 166.0 ; MS (m/z): $461\left(\mathrm{M}^{+}\right)$; Elemental Analysis: C, 57.99; H, $4.28 ; \mathrm{N}, 16.25 \%$.

2. 13. Physical constant and characterization of $2-(1 \mathrm{H}-$ benzo[d]imidazole-2-ylthio)-N-(4(1-(2-(4-hydroxybenzoyl)hydrazono)ethyl)phenyl) acetamide (5l)

Yield: $65 \%$; M.P.: $224-226{ }^{\circ} \mathrm{C}$; $1 \mathrm{H}$ NMR (400 MHz, DMSO- $\delta 6, \delta$, ppm): $2.22(3 \mathrm{H}$, s, $\left.\mathrm{CH}_{3}\right), 5.35(1 \mathrm{H}, \mathrm{s},-\mathrm{OH}), 4.08\left(2 \mathrm{H}, \mathrm{s},-\mathrm{CH}_{2}\right), 6.80-7.74(12 \mathrm{H}, \mathrm{d}, \mathrm{Ar}-\mathrm{H}), 4.98-7.00(2 \mathrm{H}, \mathrm{s},-$ $\mathrm{NH})$; 13C NMR (400 MHz, DMSO- $\delta 6, \delta, \mathrm{ppm}): 16.8,39.0,113.2,113.2,116.8,116.8$, $120.4,120.4,121.5,121.5,125.2,125.2,128.4,128.4,131.4,132.3,132.3,137.4,137.4$, 139.5, 146.9, 146.9, 163.6, 166.7; MS (m/z): $459\left(\mathrm{M}^{+}\right)$; Elemental Analysis: C, 58.62; H, $4.28 ; \mathrm{N}, 16.42 \%$.

\section{RESULTS AND DISCUSSION}

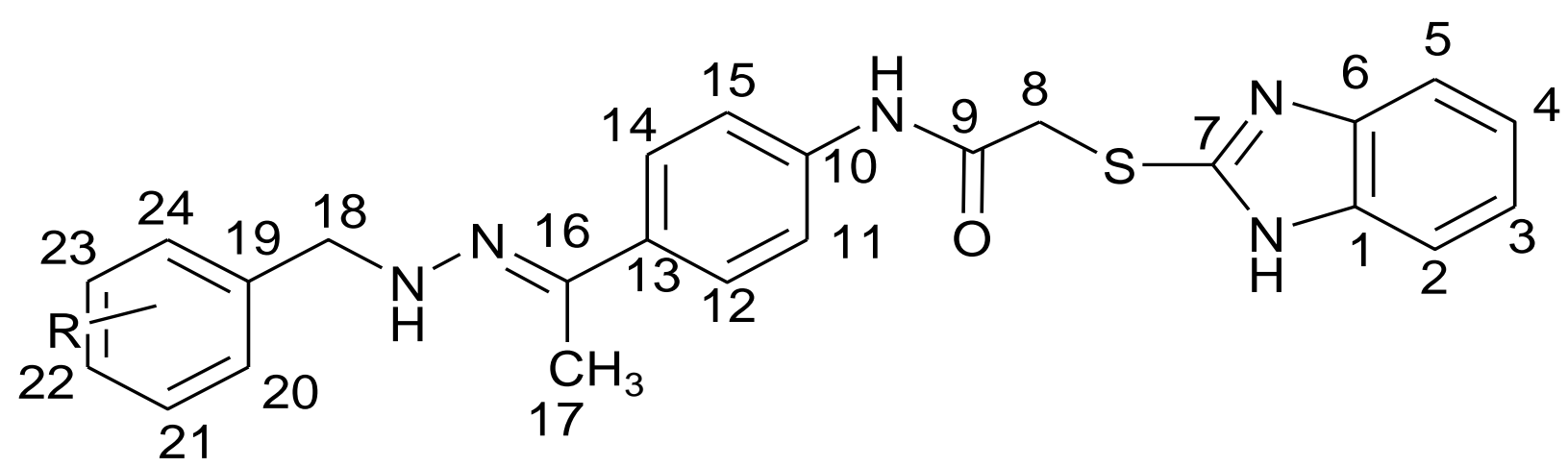

Fig. 1. Compound 5a-1. 
Compounds $5 \mathrm{a}-1$ has not been reported previously confirmed by using the Scifinder search. The structures of all compounds were confirmed by the FT-IR, ${ }^{1} \mathrm{H} \mathrm{NM},{ }^{13} \mathrm{C}$ NMR, MS and CHN analyzer. IR spectral features provide valuable information regarding the nature of functional group attached. In order to study the bonding mode of compound 4 to the compound $5 \mathrm{a}-1$, the IR spectrum of compound 4 was compared with the spectra of compound $5 \mathrm{a}-1$. Considerable differences to be expected were observed.

The structures of the final compounds $5 \mathrm{a}-1$ were established by their spectral analysis. Using compound 5a as a representative example, its FTIR spectrum of 5a showed the most relevant peaks of benzimidaole-acetylphenyl ring. Stretching vibration at $3227 \mathrm{~cm}^{-1}$ indicates that compound containing a secondary amine. The vibration at $1582 \mathrm{~cm}-1$ and $3049 \mathrm{~cm}-1$ over the range showed intensity absorption peaks corresponding to aromatic $\mathrm{C}-\mathrm{H}$ stretching vibrations. The absorption peaks at $1685 \mathrm{~cm}-1$ is due to the carbonyl groups present structure.

${ }^{1} \mathrm{H}$ NMR It has been observed from the chemical structure of compound that C-11,C-12, $\mathrm{C}-14$ and $\mathrm{C}-15$ are pairs of chemically equivalent protons which appear at $\delta=7.69 \mathrm{ppm}$ and $\delta$ $=7.77 \mathrm{ppm}$ indicates that this all four carbon are presence in same aromatic ring. As well as C-2, C-3, C-4 and C-5 are pairs of chemically equivalent protons which appeared at $\delta=$ $7.24 \mathrm{ppm}$ and $\delta=7.55 \mathrm{ppm}$ value indicates that benzimidazole ring. C-20, C-21, C-22, C-23 and $\mathrm{C}-24$ are also pairs of chemically equivalent protons which indicates that this all carbon presence of aromatic ring. The protons attached at $\mathrm{C}-8$ appeared as a singlet at $\delta=3.97 \mathrm{ppm}$ due to sulfur and C-9 carbonyl group atmosphere. Carbon C-17 contains a proton gives singlet at $\delta=0.93 \mathrm{ppm}$ indicates that azomethine group present in the structure. The mass spectrum of 5 a showed a molecular ion peak at $\mathrm{m} / \mathrm{z} 443(\mathrm{M}+1)$, which is in agreement with its proposed structure.

Minimum Inhibitory Concentration for bacteria $(\mathrm{MICb})$ of all the newly synthesized compounds was determined for their in vitro antimicrobial activity against different bacterial and fungal strains by the conventional broth-dilution method [15] using standard drugs. The results of antimicrobial studies are presented in Table 1.

Intermediates 2 and 4 showed poor antimicrobial activity against all tested bacterial and fungal strains as compared to final derivatives $5 \mathrm{a}-1$. Newly synthesized derivatives showed improved antibacterial activity compared to antifungal activity. Compounds $5 \mathrm{~b}, 5 \mathrm{c}$ and $5 \mathrm{~g}$ were found to be highly active against all the bacterial strains, showing inhibition in the range of $12.5-100 \mathrm{mg} / \mathrm{ml}$. Among them, compounds $5 \mathrm{c}$ emerged as the most effective antibacterial agents with a 2 to 4 -fold higher MIC $(25-50 \mathrm{mg} / \mathrm{ml})$ than the reference drug Chloramphenicol. Compounds $5 \mathrm{~b}$ and $5 \mathrm{~g}$ exhibited comparable antibacterial activity with MIC values of 25-100 $\mathrm{mg} / \mathrm{ml}$.

Compounds $5 \mathrm{~b}$ and $5 \mathrm{~h}$ substituted with inductively electron withdrawing nitro and chloro groups respectively, at the meta and para position showed the highest antibacterial activity $(\mathrm{NO} 2>\mathrm{Cl})$. The presence of lipophilic substituent at the meta position of phenyl ring provided an optimistic influence on the antibacterial activity. The presence of electron donating groups on the phenyl ring resulted in a significant decrease in antimicrobial activity of compounds $5 \mathrm{~d}, 5 \mathrm{e}, 5 \mathrm{f}, 5 \mathrm{j}, 5 \mathrm{k}$ and 51 .

From these results, it can be observed that the antibacterial activity was considerably affected by the substitution pattern on the phenyl ring. Further, the results of the antifungal activity indicated that compound $5 \mathrm{~g}$ endowed with chlorine emerged as the most effective antifungal agent and showed an MIC in the range of $25-62.5 \mathrm{mg} / \mathrm{ml}$ against three fungal strains using ketoconazole as a positive control. 
Table 1. Antimicrobial screening results of compounds 2,4 and $\mathbf{5 a}-\mathbf{k}$.

\begin{tabular}{|c|c|c|c|c|c|c|c|c|}
\hline \multirow[t]{3}{*}{ Entry } & \multirow[t]{3}{*}{$\mathbf{R}$} & \multicolumn{7}{|c|}{ Minimum inhibitory concentration (MIC) $\mu \mathrm{g} / \mathrm{mL} \pm \mathrm{SD}$} \\
\hline & & \multicolumn{2}{|c|}{$\frac{\text { Gram positive }}{\underline{\text { bacteria }}}$} & \multicolumn{2}{|c|}{$\frac{\text { Gram negative }}{\text { bacteria }}$} & \multicolumn{3}{|c|}{ Fungi } \\
\hline & & $\begin{array}{c}\text { S. } \\
\text { aureus }\end{array}$ & $\begin{array}{c}\text { S. } \\
\text { pyogenes }\end{array}$ & $\begin{array}{l}\text { E. } \\
\text { coli }\end{array}$ & $\begin{array}{c}\mathrm{P} . \\
\text { aeruginosa }\end{array}$ & $\begin{array}{c}\mathrm{C} . \\
\text { albicans }\end{array}$ & $\begin{array}{c}\text { A. } \\
\text { niger }\end{array}$ & $\begin{array}{c}\text { A. } \\
\text { clavatus }\end{array}$ \\
\hline 2 & - & $>1000$ & $>1000$ & $>1000$ & $>1000$ & $>1000$ & $>1000$ & $>1000$ \\
\hline 4 & - & $500 \pm 2 *$ & $500 \pm 1 *$ & $500 \pm 2 *$ & $1000 \pm 2 *$ & $1000 \pm 2 *$ & $1000 \pm 2 *$ & $>1000$ \\
\hline 5a & $-\mathrm{H}$ & $250 \pm 4 *$ & $250 \pm 4 *$ & $500 \pm 3^{*}$ & $500 \pm 3 *$ & $200 \pm 2 *$ & $100 \pm 3 *$ & $200 \pm 2 *$ \\
\hline 5b & $3-\mathrm{NO}_{2}$ & $50 \pm 4 *$ & $50 \pm 3 *$ & $25 \pm 2 *$ & $50 \pm 2 *$ & $100 \pm 2 *$ & $500 \pm 2^{*}$ & $1000 \pm 3^{*}$ \\
\hline $5 c$ & $4-\mathrm{NO}_{2}$ & $25 \pm 3 *$ & $25 \pm 3 *$ & $12.5 \pm 2 *$ & $25 \pm 3 *$ & $100 \pm 3 *$ & $200 \pm 3 *$ & $500 \pm 2 *$ \\
\hline $5 d$ & $3-\mathrm{CH}_{3}$ & $500 \pm 2 *$ & $500 \pm 4 *$ & $500 \pm 3^{*}$ & $500 \pm 3^{*}$ & $500 \pm 3^{*}$ & $500 \pm 2 *$ & $500 \pm 2 *$ \\
\hline $5 e$ & $4-\mathrm{CH}_{3}$ & $500 \pm 3 *$ & $500 \pm 3 *$ & $250 \pm 3 *$ & $250 \pm 3 *$ & $500 \pm 3 *$ & $500 \pm 3 *$ & $1000 \pm 3 *$ \\
\hline $5 f$ & $4-\mathrm{OCH}_{3}$ & $500 \pm 2 *$ & $500 \pm 4 *$ & $500 \pm 3^{*}$ & $500 \pm 3^{*}$ & $250 \pm 2 *$ & $500 \pm 2 *$ & $500 \pm 2 *$ \\
\hline $5 g$ & $2-\mathrm{Cl}$ & $25 \pm 2 *$ & $50 \pm 3 *$ & $50 \pm 2 *$ & $100 \pm 3 *$ & $25 \pm 2 *$ & $50 \pm 2 *$ & $62.5 \pm 3 *$ \\
\hline $5 \mathrm{~h}$ & $3-\mathrm{Cl}$ & $100 \pm 3 *$ & $100 \pm 3 *$ & $250 \pm 3 *$ & $250 \pm 3 *$ & $100 \pm 2 *$ & $100 \pm 2 *$ & $200 \pm 3 *$ \\
\hline $5 i$ & $4-\mathrm{Cl}$ & $200 \pm 3 *$ & $100 \pm 3 *$ & $250 \pm 2 *$ & $250 \pm 2 *$ & $100 \pm 2 *$ & $100 \pm 3^{*}$ & $200 \pm 3 *$ \\
\hline $5 \mathbf{j}$ & $2-\mathrm{OH}$ & $200 \pm 2 *$ & $200 \pm 3 *$ & $100 \pm 2 *$ & $150 \pm 3 *$ & $500 \pm 3 *$ & $500 \pm 2 *$ & $500 \pm 3 *$ \\
\hline $5 \mathbf{k}$ & $3-\mathrm{OH}$ & $200 \pm 3 *$ & $500 \pm 3 *$ & $150 \pm 3^{*}$ & $250 \pm 2 *$ & $500 \pm 3^{*}$ & $1000 \pm 2 *$ & $1000 \pm 2 *$ \\
\hline 51 & $4-\mathrm{OH}$ & $500 \pm 2 *$ & $500 \pm 2 *$ & $250 \pm 2 *$ & $500 \pm 2 *$ & $500 \pm 3^{*}$ & $1000 \pm 2 *$ & $100 \pm 2 *$ \\
\hline $\begin{array}{l}\text { Chlora- } \\
\text { mphenicol }\end{array}$ & & $50 \pm 2$ & $50 \pm 2$ & $50 \pm 2$ & $50 \pm 2$ & - & - & - \\
\hline $\begin{array}{l}\text { Ketoco- } \\
\text { nazole }\end{array}$ & & - & - & - & - & $50 \pm 2.0$ & $50 \pm 0.5$ & $50 \pm 1.4$ \\
\hline
\end{tabular}

SD Standard deviation

$* \mathrm{P} \leq 0.0001$

\section{CONCLUSION}

We have accomplished the synthesis of new derivatives of benzimidazole 5a-k having Azomethine group with the hope of generating new bioactive molecules that could be useful as potent antimicrobial agents. A series of compounds when substituted by electronwithdrawing group like $\mathrm{NO}_{2}$ and $\mathrm{Cl}$ enhances the antimicrobial activity when present on aromatic ring. Among the twelve newer derivatives, analogs 5b, 5c and $5 \mathrm{~g}$ possessing electron withdrawing atom/group such as Nitro and Chloro at the meta or para position were identified as the most potent antibacterial agents and compound $5 \mathrm{~h}$ was found to be the most effective antifungal agent. The results lead us to further studies to acquire more information about structure-activity relationships are in progress in our laboratory.

\section{References}

[1] Camacho J., Barazarte A., Gamboa N., Rodrigues J., Rojas R., Vaisberg A., Gilman R., Charris J., Bioorg. \& Med. Chem. 19 (2011) 2023-2029. 
[2] Porcari A.R., Devivar R.V., Kucera L.S., Drach J.C., Townsend L.B., J Med Chem 41 (1998) 1252-1262.

[3] Victor F., Brown T.J., Campanale K., Heinz B.A., Shirpley L.A., Su S.K., Tang J., Vance L.M., Spitzer W., J Med Chem 40 (1997) 1511-1518.

[4] Ansari K.F., Lal C., Eur. J. Med. Chem. 44 (2009) 4028-4033.

[5] Hosamani K.S., Shingalapur R.V., Rangappa S., Hugar M.H., Eur J Med Chem 45 (2010) 1753-1759.

[6] Patil A., Ganguly S., Surana S., Rasayan J Chem. 1 (2008) 447-460.

[7] Deep Joshi, Kalpesh Parikh, Med. Chem. Res. 22 (2012) 3688-3697.

[8] Kalpesh S. Parikh and Sandip P. Vyas, Archives of Applied Science Res. 4 (2012) 15781580 .

[9] Grocer, H., Kus, C., Boykin, D.W., Yildiz, S., Altanlar, N. Synthesis and Anti-fungal Properties of Some Benzimidazole Derivatives. Bioorg. Med. Chem.10 (2002) 25892596.

[10] Nicholson R.M., Murphy J.R., Dearden J.R., Journal of Pharmacy and Pharmacology 34 (1982) 106-111.

[11] Fang B., Zhou C.H., Rao X.C., Eur. J. Med. Chem. 45 (2010) 4388-4398.

[12] E. F. Magomedova, V. V. Pinyaskin, A. Sh. Aminova, Pharma. Chem. J. 41 (2007) 474-475.

[13] Yadav S., Kumar P., Clercq E.D., Balzarini J., Pannecouque C., Dewan S.K., Narasimhan B., Eur. J. Med. Chem. 45 (2010) 5985-5997.

[14] John Maria Xavier, M. Arockia Raj, J. Margaret Marie, Journal of Chemical and Pharmaceutical Research 4 (2012) 669-672.

[15] P.C. Hannan, Vet. Res. 31 (2000) 373-395. 